\title{
Developing a "toolkit" to measure implementation of concurrent palliative care in rural community cancer centers
}

\author{
LISA ZUBKOFF, PH.D., ${ }^{1}$ J. NICHOLAS DIONNE-ODOM, PH.D., R.N., ${ }^{2}$ MARIA PISU, PH.D., ${ }^{3}$ \\ DILIP BABU, M.D., ${ }^{3}$ IMATULLAH AKYAR, PH.D., R.N., ${ }^{4}$ TASHA SMITH, PH.D., ${ }^{5}$ \\ GISELLA A. MANCARELLA, M.P.H., ${ }^{2}$ LUCY GANSAUER, R.N., M.s.N., O.C.N., C.C.R.P., \\ MARGARET MURRAY SULLIVAN, M.S., ${ }^{7}$ KEITH M. SWETZ, M.D., M.A., 8 \\ ANDRES AZUERO, PH.D., ${ }^{2}$ AND MARIE A. BAKITAS, D.N.SC., C.R.N.P. ${ }^{2,3}$ \\ ${ }^{1}$ Health Science Specialist, White River Junction VA Medical Center, White River Junction, Vermont, USA \\ ${ }^{2}$ School of Nursing, University of Alabama at Birmingham, Birmingham, Alabama, USA \\ ${ }^{3}$ School of Medicine, University of Alabama at Birmingham, Birmingham, Alabama, USA \\ ${ }^{4}$ School of Nursing, Hacettepe University, Ankara, Turkey \\ ${ }^{5}$ Division of Hematology and Oncology, University of Alabama at Birmingham, Birmingham, Alabama, USA \\ ${ }^{6}$ Gibbs Cancer Center and Research Institute, Spartanburg, South Carolina, USA \\ ${ }^{7}$ University of South Alabama Mitchell Cancer Institute, Mobile, Alabama, USA \\ ${ }^{8}$ Birmingham VA Medical Center, Birmingham, Alabama, USA \\ (RECEIved February 8, 2017; ACCEPTEd March 23, 2017)
}

\begin{abstract}
Objective: Despite national guidelines recommending early concurrent palliative care for individuals newly diagnosed with metastatic cancer, few community cancer centers, especially those in underserved rural areas do so. We are implementing an early concurrent palliative care model, ENABLE (Educate, Nurture, Advise, Before Life Ends) in four, rural-serving community cancer centers. Our objective was to develop a "toolkit" to assist community cancer centers that wish to integrate early palliative care for patients with newly diagnosed advanced cancer and their family caregivers.

Method: Guided by the RE-AIM (Reach, Effectiveness-Adoption, Implementation, Maintenance) framework, we undertook an instrument-development process based on the literature, expert and site stakeholder review and feedback, and pilot testing during site visits.

Results: We developed four instruments to measure ENABLE implementation: (1) the ENABLE RE-AIM Self-Assessment Tool to assess reach, adoption, implementation, and maintenance; (2) the ENABLE General Organizational Index to assess institutional implementation; (3) an Implementation Costs Tool; and (4) an Oncology Clinicians' Perceptions of Early Concurrent Oncology Palliative Care survey.

Significance of results: We developed four measures to determine early palliative care implementation. These measures have been pilot-tested, and will be integrated into a comprehensive "toolkit" to assist community cancer centers to measure implementation outcomes. We describe the lessons learned and recommend strategies for promoting long-term program sustainability.
\end{abstract}

KEYWORDS: Palliative care, Oncology, Implementation, Measure development

Address correspondence and reprint requests to: Lisa Zubkoff, White River Junction VA Medical Center, 215 North Main Street, 10A4E, White River Junction, Vermont 05009. E-mail: Lisa. Zubkoff@va.gov.

\section{INTRODUCTION}

The evidence to support the benefit of palliative care in oncology has been mounting, with demonstrated improvements in patient and caregiver quality of 
life and depression, patient symptoms, caregiver burden, patient survival, hospice use, and patient and family satisfaction (Temel et al., 2010; Greer et al., 2012; Zimmermann et al., 2014; Bakitas et al., 2009a; Davis et al., 2015; Bakitas et al., 2009b; 2015; Dionne-Odom et al., 2015). Despite professional recommendations and a growing evidence base, the level of integration of early concurrent oncologic palliative care in advanced cancer remains poor. This is true even in health systems with established palliative care teams (Wentlandt et al., 2012; Smith et al., 2012). While most U.S. hospitals with more than 300 beds report providing palliative care services, "substantial pockets" of limited availability continue to exist, especially in geographically isolated regions (e.g., rural and local community settings) (Morrison \& Meier, 2015).

ENABLE (Educate, Nurture, Advise, Before Life Ends) (Bakitas et al., 2009a; 2009b; Dionne-Odom et al., 2016a; 2016b; 2015) is an early concurrent oncology palliative care telehealth model that was designed to be seamlessly integrated with usual oncology care at the time of an advanced cancer diagnosis, especially for rural-dwelling patients. ENABLE includes (1) an initial in-person palliative care consultation and (2) a series of structured phone-based coaching sessions for patients and their family caregivers. Trained palliative care nurse coaches facilitate the phone sessions using a guidebook called "Charting Your Course" (CYC). The topical areas include problem solving, symptom management, self-care, communication and decision-making, and life outlook and review (Steinhauser et al., 2008; 2009). After completion of phone sessions, nurse coaches follow-up monthly with patients and caregivers to reinforce prior content and address new issues. Two randomized controlled trials of ENABLE have demonstrated benefits related to patients' symptoms, mood, and survival and caregivers' mood and burden (Bakitas et al., 2009b; 2015; Dionne-Odom et al., 2015).

Recognizing the limited uptake of early palliative care, we developed a four-year study funded by the American Cancer Society to implement ENABLE in four rural-serving community cancer centers in Alabama and South Carolina. Our study has been guided by the RE-AIM (reach, effectiveness, adoption, implementation, and maintenance), a widely recognized implementation framework to evaluate the success and public health effectiveness of translating evidence-based interventions into real-world practice (Glasgow et al., 2001; National Cancer Institute, 2016). During the implementation process, we recognized a gap in the form of a lack of tools to measure whether implementation had actually taken place. This paper describes the study's toolkit development and testing phases. We believe that this is the first time that the RE-AIM framework has been adapted to measure implementation of early palliative care.

\section{METHOD}

Our study aims were: (1) to assess palliative care practices and prepare cancer centers for organizational change; (2) to tailor and implement ENABLE at each site; and (3) to evaluate ENABLE implementation using the RE-AIM framework. A virtual learning community implementation model was employed to foster shared learning, knowledge, and peer support among participating sites. A "learning community," similar to a learning collaborative, is a network of individuals or organizations with shared goals and attitudes who provide peer support and regularly communicate to promote collaboration, learning, sharing of knowledge, and who make a long-term commitment to sustainability (Bond et al., 2016).

\section{Setting and Subjects}

Four rural-serving community cancer centers participated in this implementation study: Gibbs Cancer Center (Spartanburg, South Carolina); the University of South Alabama Mitchell Cancer Institute (Mobile, Alabama); the Birmingham Veterans Affairs Medical Center; and the University of Alabama at Birmingham (UAB) Division of Gynecologic Oncology. The institutional review board (IRB) of the UAB Coordinating Center and those of the other participating organizations approved this study.

\section{RESULTS}

We describe the relevant background and rationale, development/testing, domains/items, and scoring for the four measures developed for the implementation toolkit.

\section{The ENABLE RE-AIM Self-Assessment Tool}

\section{Background and Rationale}

The RE-AIM domains of "reach," "effectiveness," "adoption," "implementation," and "maintenance" are critically important in evaluating implementation of any new evidence-based intervention (Glasgow et al., 2001; National Cancer Institute, 2016). RE-AIM specifically evaluates a new program's impact on population health compared to effectiveness testing of participant outcomes under ideal research conditions. Interventions must "reach" a target population who are able and willing to participate. It must be feasible for healthcare institutions and clinicians 
to initiate and "adopt" the program in practice settings with existing resources, personnel, and levels of expertise. Programs must also be able to "implement" the program's essential elements as tested in the research setting. Finally, programs must be "maintained" at the individual and institution/community levels for as long as they are relevant. These five domains interact to determine the overall impact of the population-based program (Gaglio \& Glasgow, 2012). A program that performs poorly on one or two RE-AIM components may have low overall public health impact.

\section{Development and Testing}

We adapted the RE-AIM framework into open and closed items to evaluate community cancer centers' implementation of ENABLE. The tool was conceived as a way to annually evaluate institutions' goals, strengths, challenges, and benchmarks for success. The tool is not currently designed as a scale instrument (i.e., to tabulate numeric scores that assess the level of an underlying construct). Two coordinating center investigators (L.Z., J.N.D.O.) reviewed RE-AIM framework references and drafted items. Participating implementation site investigators reviewed tool drafts and provided written and verbal feedback to enhance face and content validity. The site principal investigators (PIs) (i.e., administrator or physician champion) received the finalized draft of the tool by email to complete and return prior to the baseline site visit (two to three months before formally rolling out ENABLE). Coordinating center staff reviewed responses with site personnel during individual and group interviews with site investigators, senior leadership (e.g., department chairs, hospital administrators), administrative support personnel, clinicians performing in-person palliative care assessments, and clinicians delivering ENABLE CYC sessions. Following the site visit, RE-AIM responses and notes were entered into a research electronic data capture (REDCap) database. Site visit interviews and focus groups were recorded and notes were taken during all interviews. Qualitative analysis will be performed after final site visits.

\section{Domains/Items}

The ENABLE RE-AIM Self-Assessment Tool (see Table 1) comprises 50 open and closed response items that assess "reach" (21 items), "adoption" (11 items), "implementation" (14 items), and "maintenance" (4 items). "Reach" items assess the number or proportion and representativeness of program participants. Program "effectiveness" for patient and caregiver is not assessed by this tool but rather by several validated instruments utilized in prior studies to examine quality of life, symptoms, and mood (Tables 2A and 2B) (Zimet et al., 1990; Oxman \& Hull, 2001; Glasgow et al., 2005; Schmittdiel et al., 2008; Steinhauser et al., 2004; Lyons et al., 2009; Bjelland et al., 2002; Montgomery et al., 1985; 2000; Bakas et al., 2006). "Adoption" items evaluate the number, proportion, and representativeness of settings and institutional staff who support and deliver the program. "Implementation" items assess the site's fidelity to the essential elements of the ENABLE intervention as it was tested in randomized controlled trials. "Maintenance" items examine the extent to which the ENABLE program has become part of the institution's routine organizational practices and policies (National Cancer Institute, 2016).

\section{Scoring}

Changes to and trajectories of quantitative item responses within each RE-AIM domain will be assessed at each site visit (baseline, years 1, 2, and 3). For example, to measure "reach" we will calculate the numerator/denominator ratio at each timepoint to capture change over time. We will then examine qualitative comments for additional insights that can help explain the quantitative trends over time. This mixed-methods approach will be used for each RE-AIM self-assessment domain. At the conclusion of the study, these results will be shared with sites, and we will conduct a final assessment of tool usefulness and incorporate feedback and modify the tool, for inclusion in the final toolkit.

\section{The ENABLE General Organizational Index (GOI-ENABLE)}

\section{Background and Rationale}

This tool assesses institutional "readiness," including infrastructure support, supervision, and adequately trained personnel. Originally developed to facilitate implementation of mental health best practices, the General Organizational Index (GOI) assesses institutional operating characteristics among 12 domains that are essential to any evidence-based practice uptake and maintenance (Arons \& English, 2002; Dartmouth College, 2002). The rationale for this tool is that programs with strength in these areas are expected to be more successful at achieving program implementation and target outcomes. We believe that this is the first tool to formally evaluate institutional readiness for early concurrent oncology palliative care.

\section{Development and Testing}

Similar to the ENABLE RE-AIM Self-Assessment Tool, this fidelity tool provides a structured way to 
Table 1. ENABLE RE-AIM tool items

\section{Items}

REACH (\% and representativeness of individuals willing to participate)

List disease traits of target ENABLE patient population.

Past year, list patient traits that excluded them from ENABLE.

Upcoming year, changes to exclusion criteria? Describe.

Annual estimated no. of eligible patients followed by medical center.

Past year, estimated no. of eligible patients approached for ENABLE.

Upcoming year, estimated no. eligible patients to approach.

Past year, estimated no. eligible patients who participated.

Past year, estimated no. of ENABLE caregiver participants.

Upcoming year, estimated no. of eligible patients who will participate.

Upcoming year, estimated no. of caregivers who will participate.

Estimated no. of patients giving reason(s) for not participating in ENABLE: not interested, doesn't like, don't need, concern about palliative care, too ill, too busy, other.

Program included participants of diversity? Explain.

Past year, program attracted target population? $1=$ not at all to $10=$ completely confident.

Upcoming year, program will attract target population? $1=$ not at all to $10=$ completely confident.

Past year, estimated percentage of patients who completed all ENABLE? Comments about these patients.

Upcoming year, estimated percentage of patients who will complete all ENABLE? Comments about these patients.

Past year, percentage of caregivers who completed all ENABLE? Comments about these caregivers.

Upcoming year, percentage of caregivers who will complete all ENABLE? Comments about these caregivers.

Past year, barriers limited successful dyad participation? Describe.

Upcoming year, barriers will limit successful dyad participation? Describe.

How to overcome barriers.

\section{EFFECTIVENESS/EFFICACY (assessed by patient/caregiver outcome measures; see Tables 2A and 2B)}

ADOPTION (percentage and representativeness of settings and staff who deliver ENABLE).

Who had potential to refer patients/families to ENABLE?

Upcoming year, changes?

Past year, estimated no. of clinicians who could have referred.

Upcoming year, estimated no. of clinicians who will have opportunity to refer.

Past year, estimated no. of clinicians who actually referred.

Upcoming year, estimated no. of clinicians who will refer.

Reasons clinicians refer patients/families to ENABLE.

Reasons clinicians do not refer patients/families.

Past year, greatest clinician barriers to referring patients/families.

Upcoming year, greatest clinician barriers to referring.

How to overcome barriers?

\section{IMPLEMENTATION (consistency and skill by staff to deliver program elements)}

For each item below, a core element of ENABLE, were modifications made for ENABLE program? 1. none, 2. moderate, 3. completely changed or not included.

Trigger mechanism to identify individuals with advanced cancer at or near time of diagnosis.

Patients' caregivers are invited to participate in ENABLE.

Patients referred to in-person palliative care assessment.

Site's CYC manual includes core topics (creativity, optimism, planning and expert information [COPE] attitude/problem solving; symptom management/self-care; communication, support and decision making; life review; forgiveness; creating a legacy).

Site's program provides one-on-one coaching.

Education/coaching include core topics (COPE attitude/problem solving; symptom management/self-care; communication, support, and decision making; life review (patient only); forgiveness (patient only); and creating a legacy (patient only).

Program provides monthly follow-up calls.

Bereavement call to caregiver after patient death.

Site team meetings occur regularly.

Site program evaluated yearly.

Standardized measures used for ENABLE quality improvement? Describe.

If not, have plans to? Describe.

List barriers faced to implementing ENABLE core elements. Describe.

Looking forward, how to address these barriers? Describe. 
Table 1. Continued

MAINTENANCE (extent site sustains program over time)

ENABLE program will be site-maintained after grant complete? $1=$ not at all to $10=$ completely confident.

Greatest challenges to continued support?

Stakeholder commitment to continue if successful? Explain.

Changes to ENABLE program after grant completion? Explain.

assess organizational capacity to implement the ENABLE program. As suggested by the original GOI developers, it is necessary to tailor the GOI for each specific evidence-based practice (Arons \& English, 2002; Dartmouth College, 2002). Thus, initial tailoring of the GOI-ENABLE was performed by one coordinating center member (J.N.D.O.), and subsequent iterations were reviewed and edited by other team members (L.Z., M.A.B.) and by participating site investigators. The GOI-ENABLE was used at the initial and subsequent site visits.

\section{Domains/Items}

As shown in Table 3, the GOI-ENABLE tool includes 12 tailored domains, including program philosophy, eligibility/identification, penetration, in-person palliative care assessment, individualized initial palliative care treatment plan, individualized follow-up, training, supervision, process monitoring, outcome monitoring, quality assurance, and choice in services provided. Each domain is rated on a face-valid 5point scale, where 1 represents no implementation and 5 represents full implementation.

\section{Scoring}

The ratings for each domain are determined by two or more external fidelity assessors who use multiple sources of information (semistructured interviews with key informants [e.g., staff, patients and caregivers]; meeting observations; and review of program materials and other documentation) to adjudicate scores. Guidance on discriminating high and low ratings is uniquely defined for each domain and based on concrete observable evidence of the practice element. Fidelity assessors independently assign initial scores for each domain and then decide final scores through discussion and consensus. In addition to individual domain scores, a total mean score is calculated. Higher scores represent a higher likelihood of successfully implementing ENABLE with high fidelity and sustainability.

At the conclusion of the study, we will graph each site's progress over time in each domain and provide feedback on domains that are still in need of strengthening. Change scores from baseline will be computed, and the association between change scores and change in total numbers of patients and caregivers participating in the ENABLE program each year will be estimated. From a tool development perspective, this type of analysis will be able to establish construct (criterion) validity. We will modify the GOI-ENABLE based on user experiences, fidelity assessors, and site feedback to produce the final version to be included in the toolkit.

\section{The ENABLE Implementation Cost Tool}

\section{Background and Rationale}

This tool was developed to collect information from sites to evaluate overall implementation costs. No such tool existed for early concurrent oncology palliative care prior to our work, so we developed an ENABLE-specific tool to capture information related to time spent by the site-specific staff to implement the program, and to participate in activities led by the study coordinating center, as well as other administrative costs.

\section{Development and Testing}

The coordinating center developed the cost tool through an iterative process among the investigators, the coordinating center economist (M.P.), and site staff. Using a template from a prior institutional project (Pisu et al., 2016), we identified potential program costs and sought site feedback on the feasibility of completing the tool. Teams pilot-tested the tool for 1-2 months before it was finalized and launched. The tool is a Microsoft Excel ${ }^{\circledR}$ spreadsheet that each site's key contact submits monthly to the coordinating center. The study coordinating center maintains cost data in REDCap. Sites either adopt the tool as developed or integrate the tool in their own participant tracking system.

\section{Domains/Items}

The ENABLE Implementation Cost Tool (Table 4) consists of three logs: the contact log, the administrative/meeting log, and the materials/costs log. Clinicians use the contact log to track time spent on patient and caregiver contacts, site meetings, and other administrative duties. A drop-down menu simplifies entry coding. The administrative and meeting 
Table 2A. Patient effectiveness questionnaires [Effectiveness, RE-AIM]

\begin{tabular}{|c|c|c|}
\hline Patient questionnaires & Descriptions & Scoring protocol \\
\hline $\begin{array}{l}\text { Patient demographics (self- } \\
\text { reported) }\end{array}$ & $\begin{array}{l}\text { Age, diagnosis, race, religion, } \\
\text { employment, education, household, } \\
\text { tobacco/alcohol }\end{array}$ & \\
\hline $\begin{array}{l}\text { Healthcare utilization-patient } \\
\quad(\text { reported at } 0,12 \text {, and } 24 \text { weeks })\end{array}$ & $\begin{array}{l}\text { Number of days in hospital, intensive care } \\
\text { unit, emergency room visits, history of } \\
\text { hospice/palliative care, advance } \\
\text { directive, do-not-resuscitate orders }\end{array}$ & \\
\hline $\begin{array}{l}\text { Multidimensional Scale of } \\
\text { Perceived Social Support } \\
\text { (MSPSS) }\end{array}$ & $\begin{array}{l}\text { Validated 12-item self-report measure to } \\
\text { assess perceived adequacy of social } \\
\text { support using a 7-point scale from } \\
\text { strongly disagree to strongly agree }\end{array}$ & $\begin{array}{l}\text { SCORING: Sum all items } \\
\text { RANGE: } 12-84 \text { (higher scores } \\
\text { indicate higher level of perceived } \\
\text { social support) }\end{array}$ \\
\hline $\begin{array}{l}\text { Patient Assessment of Chronic } \\
\text { Illness Care (PACIC) }\end{array}$ & $\begin{array}{l}\text { 20-item patient reported measure of } \\
\text { chronic illness care, health counseling } \\
\text { behaviors; consists of five chronic illness } \\
\text { care constructs: patient activation, } \\
\text { delivery system/decision support, goal } \\
\text { setting, problem solving, and follow-up/ } \\
\text { coordination }\end{array}$ & $\begin{array}{l}\text { SCORING: Sum all item scores; overall } \\
\text { PACIC is scored by averaging scores } \\
\text { across all } 20 \text { items }\end{array}$ \\
\hline $\begin{array}{l}\text { Quality of Life at the End of Life } \\
\text { (Qual-E) }\end{array}$ & $\begin{array}{l}\text { 26-item self-report measuring quality of } \\
\text { life of seriously ill patients }\end{array}$ & $\begin{array}{l}\text { SCORING: Sum all item scores } \\
\text { RANGE: } 21-205\end{array}$ \\
\hline $\begin{array}{l}\text { Functional Assessment of Chronic } \\
\text { Illness Therapy-Palliative Care } \\
\text { (FACIT-Pal) }\end{array}$ & 46 -item self-report measure & $\begin{array}{l}\text { RANGE: } 0-184 \text { for the FACIT-Pal; } 0- \\
76 \text { for the palliative scale alone, and } \\
0-132 \text { for the Trial Outcome Index } \\
\text { (TOI); higher scores indicate better } \\
\text { quality of life }\end{array}$ \\
\hline $\begin{array}{l}\text { Hospital Anxiety and Depression } \\
\text { Scale (HADS) }\end{array}$ & $\begin{array}{l}\text { 14-item self-report measure of depressive } \\
\text { and anxiety symptoms specifically } \\
\text { designed for medical patients; contains } \\
\text { only cognitive symptoms of depression } \\
\text { and anxiety, thus eliminating somatic } \\
\text { symptoms that are poor indicators of } \\
\text { psychiatric stress in the medically ill }\end{array}$ & $\begin{array}{l}\text { SCORING: Sum all item scores } \\
\text { RANGE: } 0-21 \text { for both anxiety and } \\
\text { depression }\end{array}$ \\
\hline
\end{tabular}

Table 2B. Caregiver effectiveness questionnaires [Effectiveness, RE-AIM]

\begin{tabular}{|c|c|c|}
\hline Caregiver questionnaires & Description & Scoring protocol \\
\hline $\begin{array}{l}\text { Caregiver demographics (self- } \\
\text { reported) }\end{array}$ & $\begin{array}{l}\text { Age, diagnosis, race, religion, employment, education, } \\
\text { household, tobacco/alcohol }\end{array}$ & \\
\hline $\begin{array}{l}\text { Montgomery-Borgatta } \\
\text { Caregiver Burden Scale } \\
\text { (MBCB) }\end{array}$ & $\begin{array}{l}\text { 14-item self-report measure of caregiver burden with three } \\
\text { subscales, providing scores for object burden, subjective } \\
\text { demand burden, and subjective stress burden }\end{array}$ & $\begin{array}{l}\text { Scoring Sheet } \\
\text { Objective burden: } \\
\text { range } 6-30 \\
\text { Subjective stress } \\
\text { burden: range } 4-20 \\
\text { Subjective demand } \\
\text { burden: range } 4-20\end{array}$ \\
\hline $\begin{array}{l}\text { Bakas Caregiving Outcomes } \\
\text { Scale (BCOS) }\end{array}$ & $\begin{array}{l}\text { 15-item degree of changes in life by providing care to } \\
\text { someone with cancer }\end{array}$ & $\begin{array}{l}\text { SCORING: Sum all items } \\
\text { RANGE: } 15-105\end{array}$ \\
\hline $\begin{array}{l}\text { Hospital Anxiety and } \\
\text { Depression Scale (HADS) }\end{array}$ & $\begin{array}{l}\text { 14-item self-report measure of depressive and anxiety } \\
\text { symptoms specifically designed for medical patients; } \\
\text { contains only cognitive symptoms of depression and } \\
\text { anxiety, thus eliminating somatic symptoms that are } \\
\text { poor indicators of psychiatric stress in the medically ill }\end{array}$ & \\
\hline Costs of Caregiving (COC) & $\begin{array}{l}\text { 14-item self-report measure of financial burden of } \\
\text { caregiving }\end{array}$ & \\
\hline
\end{tabular}


Table 3. General Organizational Index (GOI) ENABLE domains

\begin{tabular}{lll}
\hline \hline GOI-ENABLE domains and description & Interviews/data source & Example response options/scoring protocol*
\end{tabular}

\section{G1. Program philosophy}

Program committed to philosophy consistent with ENABLE model

\section{G2. Eligibility/client identification}

Targeted patient/caregivers screened using

standardized tools consistent with ENABLE criteria

G3. Penetration

Penetration percentage $=$ no. of clients with access to ENABLE divided by total no. of eligible clients who could benefit from ENABLE

\section{G4. Assessment}

ENABLE participants to receive in-person palliative care assessments following national consensus guidelines

G5. Individualized initial palliative care treatment plan ENABLE participants receive individualized care plan

G6. Individualized subsequent ENABLE contacts ENABLE participants' individualized care plans followed/reassessed

G7. Training

All staff delivering CYC ENABLE receive standardized training.

\section{G8. Supervision}

ENABLE coaches receive supervision from palliative care practitioner
1. ENABLE site personnel

2. ENABLE patients/caregivers

1. ENABLE site personnel

1. ENABLE leader provides estimates

1. ENABLE site personnel to complete inperson $\mathrm{PC}$ assessments

1. PC practitioners complete in-person PC assessments

2. ENABLE coaches caregivers received

3. ENABLE patients/caregivers

1. ENABLE coaches

2. ENABLE patients/caregivers

1. ENABLE site personnel

2. ENABLE coaches

3. Staff delivering in-person assessment

1. ENABLE site personnel
$1=$ ratio $\leq .20 ; 5=$ ratio $>0.80$

$1=$ no assessments or assessments nonstandardized; $5=>80 \%$ of clients receive standard assessments

$1=0-20 \%$ ENABLE-served patients/caregivers received initial plan; $5=>80 \%$ of patients

$1=$ only 1 of 5 sources shows clear understanding of ENABLE philosophy OR all sources have discrepancies; $5=$ all five sources show clear understanding and commitment to ENABLE

$1=$ estimated $20 \%$ patients/caregivers receive standard screen and/or clinic has no system to track eligibility; $5=$ estimated $>80 \%$ of patients caregivers receive screen, and clinic tracks eligibility

$1=20 \%$ of ENABLE patients/caregivers have individualized sessions; $5=>80 \%$ of ENABLE patients/caregivers have these sessions

$1=20 \%$ of ENABLE staff receive standardized training; $5=>80 \%$ of ENABLE staff receive training

$1=20 \%$ of ENABLE coaches receive structured supervision; $5=>80 \%$ of ENABLE coaches receive 
log tracks and records time spent by staff other than clinicians (site physician champion, other nursing staff, and administrators) on such program-related duties as time spent on personnel training to implement ENABLE. Finally, the materials/costs log tracks costs for any program-related teaching or marketing materials purchased.

\section{Scoring}

Salary and other cost data are added to the ENABLE Implementation Cost Tool to calculate implementation costs. We will separate time spent on research activities from actual program implementation costs. Time will be valued using average salaries of staff according to the titles associated with specific activities-that is, the costs of clinicians will be valued using their average salaries. Materials and other resources will be valued using site-specific expenses. We will calculate total and per-patient-served implementation costs by site and overall. Sensitivity analyses will be conducted to obtain a range of possible costs that vary depending on salary levels, time spent on various ENABLE activities, or different uptake of ENABLE patient activities (e.g., number of follow-up calls).

\section{Oncology Clinicians' Perceptions of Early Concurrent Palliative Oncology Care}

\section{Background and Rationale}

Despite professional guidelines not all oncology staff are familiar with or supportive of early palliative care and their perceptions may impact program integration. Because we were unable to locate tools that specifically assessed these perspectives we created this survey.

\section{Development and Testing}

Tool development began with a literature review of studies and tools related to perceptions of and referral barriers to oncology palliative care (Bradley et al., 2002; Cherny et al., 2003; Cherny \& Palliative Care Working Group of the European Society for Medical Oncology, 2011; Fox et al., 2007; Johnson et al., 2008; Metzger et al., 2013; Ogle et al., 2002; Sheetz \& Bowman, 2008; Ward et al., 2009; Wotton et al., 2005). Based on this review, a palliative care physician team member (D.B.), extracted relevant items, edited them, and developed additional items. The initial draft survey was reviewed for clarity and face validity by three team members (L.Z., J.N.D.O., M.A.B.), and a subsequent draft was reviewed by eight oncology clinicians. The oncology clinicians reviewed the draft survey using a standardized scoring rubric with a 4-point Likert-type scale, where scores 
Table 4. ENABLE Implementation Cost Tool (every month, participating site completes the cost tool to establish the cost of implementing the ENABLE program; within the tool, there are three distinct logs: the contact log, administrative activities and meeting log, and the materials/costs log)

\section{Contact log items (for staff and participants including patients and caregivers)}

Personnel name, role, annual salary

Personnel time spent in training, hours per month

Personnel time taken to enroll new participant(s), hours per month

For palliative care assessments, the number of palliative care assessments per patient (in min), average time per assessment per patient (in min), average preparation time per patient (in min), and average time for palliative care assessment documentation of post-assessment follow-up (in min)

For patient coaching, number of phone-delivered coaching sessions, number of in-person coaching sessions, average time per session (in min), average time for pre- and post-session documentation and follow-up (in min)

For caregiver coaching, number of phone-delivered coaching sessions, number of in-person coaching sessions, average time per session (in min), average time for pre- and post-session documentation and follow-up (in min)

For monthly follow-up after coaching sessions are complete, number of monthly contacts, average time per month (in min), average preparation time (in min), average time for monthly documentation or follow-up (in min)

For bereavement contact, the number of bereavement contacts, average time per contact (in min), average preparation time for contact (in min), average time for post-session documentation or follow-up (in min)

Administrative activities and meeting log items

Personnel name, role, ENABLE activity, time devoted to activity, comments, and annual salary

Materials/costs log items

Date, item, type of material, number of units purchased, and cost per unit for all purchased materials

ranged from not relevant (1) to very relevant and succinct (4) (Lynn, 1986). Items were revised based on reviewer feedback, focusing on items that received an average content validity score of 3 or greater. Once the final survey was complete, the site lead (Bakas, no. 26) sent a web-based (Qualtrics ${ }^{\circledR}$ ) link to their institution's oncology clinicians to complete online. Across all sites, 62 clinicians were invited to participate, 46 consented and responded, and 42 provided complete data (response rate $=68 \%$ ).

\section{Domains/Items}

Table 5 presents the 39 items and response options in the survey. Although the responses to some items in these sections may covary, the tool is not currently designed as a scale instrument.

\section{Scoring}

The survey item scores will be summed as a total score and will be employed to determine perceptions that are more accepting and positive about early palliative care in general. The survey will be readministered at all four sites upon study completion. This tool may offer insights into changes in perceptions for oncologists at sites implementing the ENABLE program.

\section{DISCUSSION}

To our knowledge, this is the first study to develop and pilot test a toolkit to facilitate and measure implementation of early concurrent oncology palliative care. The four instruments cover the essential aspects of program implementation as per the RE-AIM framework. The overall study goal was to develop implementation processes and tools to assist community cancer centers to implement national guidelines recommending integration of early concurrent palliative care for individuals newly diagnosed with advanced cancer. As with much of health services research, valid measures are critical to determining implementation success. The biggest challenge faced is a lack of appropriate measures to assess implementation of a palliative care program. We have made substantial progress in assessing the implementation of the ENABLE program, but there is much work that remains to be done.

As we embarked on creating the tools and implementation toolkit, there were a number of challenges and lessons learned. First, we found variations in initial site readiness. One site had limited personnel resources, including a lack of dedicated program staff and palliative care specialists, reflecting the shortage of palliative care specialists in the United States (Bui, 2012; Kamal et al., 2015; Lupu \& American Academy of Hospice and Palliative Medicine Workforce Task Force, 2010). Second, there were often increasing clinical responsibilities for clinicians who had planned to devote time to implement ENABLE. A lack of program staff and dedicated effort delayed program launch for some sites by several months compared to sites that had already identified staff champions to implement the ENABLE program. Third, our research grant provided sites with only meager start-up funds and did not cover salary 
Table 5. Oncology clinicians' perceptions of early concurrent oncology palliative care questionnaire items

For the following statements, choose the option that best reflects your level of agreement.

Clinician attitudes about palliative care (response options: $1=$ strongly disagree to $5=$ strongly agree)

A medical oncologist is the best person to manage the physical symptoms of patients with advanced cancer

A medical oncologist is the best person to manage the psychological symptoms of patients with advanced cancer

A palliative care specialist is the best person to manage the psychological symptoms of patients with advanced cancer

A palliative care specialist is the best person to manage the psychological symptoms of patients with advanced cancer

I find it professionally satisfying to care for the physical symptoms of my patients

I would rather not care for advanced cancer patients who are dying

I find it professionally satisfying to manage patients with advanced cancer who are dying

I feel emotionally exhausted by having to deal with patients with advanced cancer who are dying

I believe that it is important to stay current with the state of the science in palliative care related to oncology

I believe that it is important to have a good working relationship with the palliative care service at my facility

In general, patients with advanced cancer would benefit if palliative care were initiated earlier on in the course of their illness

I can control the symptoms of most patients with advanced cancer as effectively as a palliative care specialist can

When I discuss using palliative care services, patients and families often lose hope

I believe patients with advanced cancer should be referred to palliative care specialists prior to the end of life

Clinician feelings about palliative care specialists (response options: $1=$ strongly disagree to $5=$ strongly agree)

When I refer a patient to palliative care specialists, I feel that I lose control over management of the patient

When palliative care specialists are involved in the care of a patient, it is difficult to determine who is responsible for medical management

When I discuss referral to palliative care specialists, patients feel that nothing more can be done

Consulting with palliative care specialists adds value to the care of patients with advanced cancer

Palliative care specialists should be involved from the time of a diagnosis of advanced cancer

I sometimes worry that palliative care specialists limit the anticancer treatment options of patients with advanced cancer

When I refer patients to palliative care specialists, I get concerned that they will interfere with the patient's anticancer treatment

Patients with complex physical symptoms benefit from involvement of palliative care specialists

Patients with complex psychological symptoms benefit from involvement of palliative care specialists

Clinician comfort with and preferences for providing early concurrent oncology palliative care (response options: $1=$ strongly disagree to $5=$ strongly agree; Likert $=$ type scale response options)

I feel very comfortable with providing generalist palliative care in the following areas: cancer pain, dyspnea, nausea and vomiting, depression and anxiety, delirium, advance directives, end-of-life care, conducting family meetings, hospice referrals, existential or spiritual distress, requests for hastened death

In your dealings with patients with advanced cancer, how often do you collaborate with a palliative care specialist?

Which of the following best describes the specialist palliative care services available at your facility?

What is your preference in working with palliative care for patients newly diagnosed with advanced disease?

Approximately what percentage of your patients newly diagnosed with advanced cancer do you regularly refer to a specialist palliative care service?

Description of early concurrent oncology palliative care provided within their organization and

demographic data (response options: yes/no; list of appropriate options; open-ended responses)

Which of the following most closely described how the 2012 ASCO recommendation should be translated into practice? Site location

Are you aware of an outpatient supportive/palliative care program at your facility?

Do you refer patients to this program?

Why or why not? [open text response]

Gender

Age

Racial background

Clinical background/training

Number of years of postgraduate experience [open text response]

How many years have you practiced at your current institution? [open text response]

support. This resulted in difficulties securing adequate space, staff, and enough time to launch the program.

The UAB Coordinating Center was also greatly hampered by administrative barriers. First, there were inconsistent IRB interpretations of implementation into medical practices. The lack of a standard- ized IRB approach (Patel et al., 2013) across sites led to multiple and prolonged IRB application revisions. Given the struggle with gaining IRB approval, future work might be enhanced by standardized IRB procedures, additional institutional support, and improved clinician knowledge prior to the start of the program. Relatedly, we encountered difficulties 
with centralized data collection, as several sites had data-sharing restrictions. Data collection had to be adapted in such a manner that only deidentified data could be shared with the coordinating center. Helping to mitigate this issue was creation of a firewall-protected REDCap database (Harris et al., 2009) for all data collection.

Although we attempted to capture a diverse range of institutions, there are only four sites participating in this effort, which may limit the generalizability of the knowledge gained. Some of the instruments may be difficult to implement in other settings and may require modifications. Future use of these tools will include more geographically diverse populations and settings. We also recognize that the use of our instruments is labor-intensive, both for the research team and the sites completing the instruments. An automated electronic data collection tool would significantly reduce this burden.

Furthermore, the instruments rely on self-reported data, which may or may not be an accurate depiction of the program. This manuscript presents the development and initial use of the instruments. However, additional validation processes will be conducted upon conclusion of the study, including evaluation of the feasibility of its use in clinical practice.

\section{CONCLUSIONS}

Measuring whether program implementation and dissemination have occurred is a critical and developing area for early concurrent oncology palliative care and requires systematic collaboration among key stakeholders: patients and families, clinicians, program administrators, and implementation scientists. Study outputs advance the field by offering methods and measures when implementing early concurrent oncology palliative care. Despite these challenges, we believe that the work reported here will help community cancer centers overcome barriers to implementing early concurrent palliative care. Going forward, the remaining goals are to finalize the ENABLE Early Palliative Care Implementation Toolkit, to establish program sustainability at the four community cancer centers, and to develop a larger implementation study using the toolkit and comparing different implementation methods.

\section{ACKNOWLEDGMENTS}

We gratefully acknowledge the efforts of our partnering community cancer centers and their staff, including: Brian Bell, M.D., Steve Corso, M.D., Melissa Poulnott, L.P.N., Sara Ferguson, R.N., B.S.N., Andrew M. Fischer, M.Div., Nancy Anderson, M.Div., Noel Kinard, M.S.W., Chad Dingman, M.S.W., and Melody Carnes, Li.S.W.-C.P. at Spartan- burg Regional Medical Center; Jennifer Scalici, M.D., and Leigh Minchew, D.N.P., at Mitchell Cancer Institute; Kerri Bevis, M.D., Elizabeth Kvale, M.D., Gabrielle Rocque, M.D., and Amanda Erba, B.S.N., R.N., from the Division of Gynecologic Oncology at the University of Alabama at Birmingham; and Amos Bailey, M.D. (recently relocated to the University of Colorado in Denver), Neal Steil, M.D., Alfreida Hogan, D.N.P., and Will Callans, M.P.H., at the Birmingham Veterans Affairs Medical Center.

\section{AUTHOR DISCLOSURES}

The authors hereby declare that they have no competing financial interests to disclose.

\section{SOURCES OF SUPPORT}

This study was funded by an American Cancer Society Research Scholar Grant (no. RSG PCSM-124668; PI: Bakitas). J. Nicholas Dionne-Odom, Ph.D., R.N., has been supported by NIH/NINR (no. 1K99NR015903), the National Cancer Institute (no. 2R25CA04788824), and the National Palliative Care Research Center. Imatullah Akyar, Ph.D., R.N., was supported by The Scientific and Technological Research Council of Turkey (Tubitak).

\section{REFERENCES}

Arons, B.S. \& English, M.J. (2002a). Assertive Community Treatment: Implementation Resource Kit User's Guide. Hanover, NH: Implementing Evidence-Based Practices Project, Dartmouth College. Available from http:// www.dhs.state.mn.us/main/groups/disabilities/documents/ pub/dhs_id_027765 1.pdf.

Bakas, T., Champion, V., Perkins, S.M., et al. (2006). Psychometric testing of the revised 15-item Bakas Caregiving Outcomes Scale. Nursing Research, 55(5), 346-355.

Bakitas, M., Lyons, K.D., Hegel, M.T., et al. (2009a). The Project ENABLE II randomized controlled trial to improve palliative care for rural patients with advanced cancer: Baseline findings, methodological challenges, and solutions. Palliative \& Support Care, 7(1), 75-86. Available from https://www.ncbi.nlm.nih.gov/pmc/ articles/PMC3685415/.

Bakitas, M., Lyons, K.D., Hegel, M.T., et al. (2009b). Effects of a palliative care intervention on clinical outcomes in patients with advanced cancer: The Project ENABLE II randomized controlled trial. The Journal of the American Medical Association, 302(7), 741-749. Available from https://www.ncbi.nlm.nih.gov/pmc/articles/ PMC3657724/.

Bakitas, M.A., Tosteson, T.D., Li, Z., et al. (2015). Early versus delayed initiation of concurrent palliative oncology care: Patient outcomes in the ENABLE III randomized controlled trial. Journal of Clinical Oncology, 33(13), 1438-1445. Epub ahead of print Mar 23. Available from https://www.ncbi.nlm.nih.gov/pmc/articles/ PMC4404422/.

Bjelland, I., Dahl, A.A., Haug, T.T., et al. (2002). The validity of the Hospital Anxiety and Depression Scale: An 
updated literature review. Journal of Psychosomatic Research, 52(2), 69-77.

Bond, G.R., Drake, R.E., Becker, D.R., et al. (2016). The IPS learning community: A longitudinal study of sustainment, quality, and outcome. Psychiatric Services, 67(8), 864-869. Epub ahead of print Apr 1.

Bradley, E.H., Cramer, L.D., Bogardus, S.T., Jr., et al. (2002). Physicians' ratings of their knowledge, attitudes, and end-of-life-care practices. Academic Medicine, 77(4), 305-311.

Bui, T. (2012). Effectively training the hospice and palliative medicine physician workforce for improved end-oflife health care in the United States. The American Journal of Hospice \& Palliative Care, 29(6), 417-420. Epub ahead of print Dec 15, 2011.

Cherny, N.I. \& Palliative Care Working Group of the European Society for Medical Oncology (2011). Factors influencing the attitudes and behaviors of oncologists regarding the truthful disclosure of information to patients with advanced and incurable cancer. Psycho-Oncology, 20(12), 1269-1284. Epub ahead of print Sep 28, 2011.

Cherny, N.I., Catane, R. \& European Society of Medical Oncology Taskforce on Palliative and Supportive Care (2003). Attitudes of medical oncologists toward palliative care for patients with advanced and incurable cancer: Report on a survey by the European Society of Medical Oncology Taskforce on Palliative and Supportive Care. Cancer, 98(11), 2502-2510.

Dartmouth College (2002). General Organizational Index (GOI): Item Definitions and Scoring. Hanover, NH: Dartmouth College. Available from http://www. dartmouth.edu/ implementation/page19/page3/files/ goi_protocol_11-25-02.pdf.

Davis, M.P., Temel, J.S., Balboni, T., et al. (2015). A review of the trials which examine early integration of outpatient and home palliative care for patients with serious illnesses. Annals of Palliative Medicine, 4(3), 99-121. Available from http://apm.amegroups.com/ article/view/6813/7819.

Dionne-Odom, J.N., Azuero, A., Lyons, K.D., et al. (2015). Benefits of early versus delayed palliative care to informal family caregivers of patients with advanced cancer: Outcomes from the ENABLE III randomized controlled trial. Journal of Clinical Oncology, 33(13), 1446-1452. Epub ahead of print Mar 23. Available from https:// www.ncbi.nlm.nih.gov/pmc/articles/PMC4404423/.

Dionne-Odom, J., Azuero, A., Lyons, K., et al. (2016a). Family caregiver depressive symptom and grief outcomes from the ENABLE III randomized controlled trial. Journal of Pain and Symptom Management, 52(3), 378-385. Epub ahead of print Jun 3.

Dionne-Odom, J., Lyons, K., Akyar, I., et al. (2016b). Coaching family caregivers to become better problem solvers when caring for persons with advanced cancer. Journal of Social Work in End-of-Life and Palliative Care, 12(1-2), 63-81.

Fox, E., Myers, S. \& Pearlman, R.A. (2007). Ethics consultation in United States hospitals: A national survey. The American Journal of Bioethics, 7(2), 13-25.

Gaglio, B. \& Glasgow, R. (2012). Evaluation approaches for dissemination and implementation research. In Dissemination and Implementation Research in Health: Translating Science to Practice. R.C. Brownson et al. (eds.), pp. 327-356. New York: Oxford University Press.

Glasgow, R.E., McKay, H.G., Piette, J.D., et al. (2001). The RE-AIM framework for evaluating interventions: What can it tell us about approaches to chronic illness management? Patient Education and Counseling, 44(2), $119-127$.

Glasgow, R.E., Wagner, E.H., Schaefer, J., et al. (2005). Development and validation of the Patient Assessment of Chronic Illness Care (PACIC). Medical Care, 43(5), 436-444.

Greer, J.A., Pirl, W.F., Jackson, V.A., et al. (2012). Effect of early palliative care on chemotherapy use and end-oflife care in patients with metastatic non-small-cell lung cancer. Journal of Clinical Oncology, 30(4), 394-400.

Harris, P.A., Taylor, R., Thielke, R., et al. (2009). Research electronic data capture (REDCap): A metadata-driven methodology and workflow process for providing translational research informatics support. Journal of Biomedical Informatics, 42(2), 377-381.

Johnson, C.E., Girgis, A., Paul, C.L., et al. (2008). Cancer specialists' palliative care referral practices and perceptions: Results of a national survey. Palliative Medicine, $22(1), 51-57$.

Kamal, A.H., Maguire, J.M. \& Meier, D.E. (2015). Evolving the palliative care workforce to provide responsive, serious illness care. Annals of Internal Medicine, 163(8), 637-638. Epub ahead of print Aug 11.

Lupu, D. \& American Academy of Hospice and Palliative Medicine Workforce Task Force (2010). Estimate of current hospice and palliative medicine physician workforce shortage. Journal of Pain and Symptom Management, 40(6), 899-911.

Lynn, M.R. (1986). Determination and quantification of content validity. Nursing Research, 35(6), 382-385.

Lyons, K.D., Bakitas, M., Hegel, M.T., et al. (2009). Reliability and validity of the Functional Assessment of Chronic Illness Therapy-Palliative Care (FACITPal) scale. Journal of Pain and Symptom Management, 37(1), 23-32. Epub ahead of print May 27. Available from https://www.ncbi.nlm.nih.gov/pmc/articles/ PMC2746408/.

Metzger, M., Norton, S.A., Quinn, J.R., et al. (2013). Patient and family members' perceptions of palliative care in heart failure. Heart \& Lung, 42(2), 112-119. Epub ahead of print Dec 17, 2012. Available from https://www.ncbi.nlm.nih.gov/pmc/articles/PMC3593951/.

Montgomery, R., Gonyea, J. \& Hooyman, N. (1985). Caregiving and the experience of subjective and objective burden. Family Relations, 34(1), 19-26.

Montgomery, R., Borgatta, E. \& Borgatta, M. (2000). Societal and family change in the burden of care. In Who Should Care for the elderly? An East-West Value Divide. B. Lui \& H. Kendig (eds.), pp. 27-54. River Edge, NJ: World Scientific.

Morrison, R.S. \& Meier, D.E. (2015). America's Care of Serious Illness: 2015 State-by-State Report Card on Access to Palliative Care in Our Nation's Hospitals. New York: Center to Advance Palliative Care.

National Cancer Institute (2016). What Is RE-AIM? Blacksburg, VA: RE-AIM.org, Virginia Tech. Available from https://cancercontrol.cancer.gov/IS/.

Ogle, K.S., Mavis, B. \& Wyatt, G.K. (2002). Physicians and hospice care: Attitudes, knowledge, and referrals. Journal of Palliative Medicine, 5(1), 85-92.

Oxman, T.E. \& Hull, J.G. (2001). Social support and treatment response in older depressed primary care patients. The Journals of Gerontology. Series B: Psychological Sciences and Social Sciences, 56(1), 35-45.

Patel, D.I., Stevens, K.R. \& Puga, F. (2013). Variations in institutional review board approval in the 
implementation of an improvement research study. Nursing Research and Practice, 2013. Epub ahead of print Apr 23. Available from https://www.ncbi.nlm. nih.gov/pmc/articles/PMC3655662/.

Pisu, M., Meneses, K., Azuero, A., et al. (2016). Variation in resources needed to implement psychosocial support interventions for rural breast cancer survivors. Journal of Cancer Survivorship: Research and Practice, 10(2), 375-383. Epub ahead of print Sep 4, 2015.

Schmittdiel, J., Mosen, D.M., Glasgow, R.E., et al. (2008). Patient assessment of chronic illness care (PACIC) and improved patient-centered outcomes for chronic conditions. Journal of General Internal Medicine, 23(1), 77-80. Epub ahead of print Nov 21, 2007. Available from https://www.ncbi.nlm.nih.gov/pmc/articles/ PMC2173922/.

Sheetz, M.J. \& Bowman, M.A. (2008). Pediatric palliative care: An assessment of physicians' confidence in skills, desire for training, and willingness to refer for end-oflife care. The American Journal of Hospice \& Palliative Care, 25(2), 100-105.

Smith, C.B., Nelson, J.E., Berman, A.R., et al. (2012). Lung cancer physicians' referral practices for palliative care consultation. Annals of Oncology, 23(2), 382-387. Epub ahead of print Jul 29, 2011. Available from https:// www.ncbi.nlm.nih.gov/pmc/articles/PMC3265546/.

Steinhauser, K.E., Clipp, E.C., Bosworth, H.B., et al. (2004). Measuring quality of life at the end of life: Validation of the QUAL-E. Palliative \& Supportive Care, $2(1), 3-14$.

Steinhauser, K.E., Alexander, S.C., Byock, I.R., et al. (2008). Do preparation and life completion discussions improve functioning and quality of life in seriously ill patients? Pilot randomized control trial. Journal of Palliative Medicine, 11(9), 1234-1240.

Steinhauser, K.E., Alexander, S.C., Byock, I.R., et al. (2009). Seriously ill patients' discussions of preparation and life completion: An intervention to assist with transition at the end of life. Palliative \& Supportive Care, 7(4), 393-404.

Temel, J.S., Greer, J.A., Muzikansky, A., et al. (2010). Early palliative care for patients with metastatic non-smallcell lung cancer. The New England Journal of Medicine, 363(8), 733-742. Available from http://www.nejm.org/ doi/pdf/10.1056/NEJMoa1000678.

Ward, A.M., Agar, M. \& Koczwara, B. (2009). Collaborating or co-existing: A survey of attitudes of medical oncologists toward specialist palliative care. Palliative Medicine, 23(8), 698-707. Epub ahead of print Oct 13.

Wentlandt, K., Krzyzanowska, M.K., Swami, N., et al. (2012). Referral practices of oncologists to specialized palliative care. Journal of Clinical Oncology, 30(35), 4380-4386. Epub ahead of print Oct 29.

Wotton, K., Borbasi, S. \& Redden, M. (2005). When all else has failed: Nurses' perception of factors influencing palliative care for patients with end-stage heart failure. Journal of Cardiovascular Nursing, 20(1), $18-25$.

Zimet, G.D., Powell, S.S., Farley, G.K., et al. (1990). Psychometric characteristics of the Multidimensional Scale of Perceived Social Support. Journal of Personality Assessment, 55(3-4), 610-617.

Zimmermann, C., Swami, N., Krzyzanowska, M., et al. (2014). Early palliative care for patients with advanced cancer: A cluster-randomised controlled trial. Lancet, 383(9930), 1721-1730. Epub ahead of print Feb 19. 